\title{
Method for Optical Analysis of Surface Structures of Lead-Acid Battery Electrodes Using a Confocal Laser Scanning Microscope
}

\author{
Jan Kabzinski, ${ }^{a, b, z}$ Heide Budde-Meiwes, ${ }^{\text {a,b }}$ Christiane Rahe, ${ }^{\text {a }}$ and Dirk Uwe Sauer ${ }^{a, b, c}$ \\ ${ }^{a}$ Electrochemical Energy Conversion and Storage Systems Group, Institute for Power Electronics and Electrical Drives \\ (ISEA), RWTH Aachen University, Aachen 52056, Germany \\ ${ }^{b}$ Jülich Aachen Research Alliance, JARA-Energy, Aachen 52066, Germany \\ ${ }^{c}$ Institute for Power Generation and Storage Systems (PGS), E.ON ERC, RWTH Aachen University, Aachen 52056, \\ Germany
}

\begin{abstract}
A method for analyzing electrode surfaces of lead-acid batteries has been developed. It provides a clear view on crystal structures. The technique employs confocal laser scanning microscopy (CLSM). The developed method allows examination of large areas $\left(1 \mathrm{~mm}^{2}\right.$ or larger) and can be applied to entire battery plates. Specimen preparation is less laborious and costly compared to scanning electron microscopy (SEM) and still gives useful results. In addition, it turned out that electrode structures vary significantly on a scale of tenth of $\mu \mathrm{m}$. This lowers the significance of a focused image e.g. from SEM. Suitable and reproducible conditions (washing duration, drying duration and drying temperature) for the examination and preparation of electrode surfaces are investigated. The proposed method has been applied to electrodes taken from automotive flooded lead-acid batteries; scans over areas have been acquired. Electrode structures after different operating conditions are presented.

(C) The Author(s) 2016. Published by ECS. This is an open access article distributed under the terms of the Creative Commons Attribution Non-Commercial No Derivatives 4.0 License (CC BY-NC-ND, http://creativecommons.org/licenses/by-nc-nd/4.0/), which permits non-commercial reuse, distribution, and reproduction in any medium, provided the original work is not changed in any way and is properly cited. For permission for commercial reuse, please email: oa@electrochem.org. [DOI: 10.1149/2.0861606jes] All rights reserved.
\end{abstract}

Manuscript submitted August 21, 2015; revised manuscript received March 4, 2016. Published March 17, 2016.

Lead-acid batteries face rising requirements for new applications in micro- and mild-hybrid vehicles as well as in electric vehicles. Investigation and understanding of the processes taking place within the batteries are necessary for further development and improvement of performance. Microscopic analyses allow the examination of electrode surfaces. The obtained results can also contribute to developing battery models on ageing processes, lifetime prediction and charge acceptance.

In the past, electrochemical atomic force microscopy (EC-AFM) and scanning electron microscopy (SEM) were used to investigate electrode surfaces of lead-acid batteries. In various publications ECAFM was employed to investigate the negative electrode surface. Yamaguchi et al. ${ }^{1}$ applied EC-AFM to an electrode based on a pure lead sheet in a study on charge acceptance. In Ref. 2 EC-AFM was used to examine an electrode consisting of a pure lead sheet in situ during cyclic voltammetry and after oxidation. Hirai et al. ${ }^{3}$ employed in situ EC-AFM to investigate the effect of expander additives on an electrode made of a pure lead sheet. Shiota et al. ${ }^{4}$ also worked with EC-AFM. It was possible to show in situ the deposition (discharging) and dissolution (charging) of lead-sulfate crystals on a lead dioxide surface.

SEM is also used in many research activities on lead-acid batteries to examine electrodes. Years ago Pavlov et al. ${ }^{5,6}$ showed SEM images of charged and discharged electrodes, as well as after a rest time at open circuit, when the recrystallization is visible. In the last years, Pavlov et al. ${ }^{7}$ employed SEM to investigate the effect of carbon additives on the negative active material. Lead-sulfate crystal size on negative and positive electrodes was studied by Takehara. ${ }^{8}$ Crystals are smaller when discharging with a higher current rate. When crystals are larger, the usable reaction surface during charging is smaller. In Ref. 9 the influence of carbon additives on the reactions of the negative active material was studied by Pavlov et al. with utilization of SEM. However, specimen preparation for SEM is more laborious and expensive compared to preparation for confocal laser scanning microscopy. The specimen is destroyed for other analysis methods, as it needs to be coated with a thin conductive layer (e.g. gold).

Instead of using EC-AFM or SEM a method for optical analysis of electrode surface structures using confocal laser scanning microscopy (CLSM) was developed. The method is simple, fast and inexpensive. CLSM allows exceeding limitations on maximum magnification of

${ }^{\text {z} E-m a i l: ~ b a t t e r i e s @ i s e a . r w t h-a a c h e n . d e ~}$ conventional optical microscopy. As CLSM acquires images in slices at different distances from the specimen it also obtains a height profile of the specimen. This is interesting for quantitative analyses of the surface structures, e.g. computing surface roughness parameters. Basically, it is possible to scan complete plates of SLI batteries. Thus, representative images of the surface structure can easily be acquired. Within the course of presented analysis it turned out, that the surface structures show strong variations on a scale of several tens of $\mu \mathrm{m}$ and can result in misleading interpretations when looking only at a specific area in the $\mu \mathrm{m}$-range. Variations on this scale typically remain unseen when using the EC-AFM or SEM method with high resolution. Therefore, it seems to be useful to combine CLSM with EC-AFM or SEM to really get a full insight into the surface structures. A further method to support CLSM images is porosity measurement as done in Ref. 10. This can give a deeper understanding of processes during charging and discharging.

In addition to electrode surfaces, profiles of electrodes can be observed by CLSM, although this has not been done yet within this work, e.g. the thickness of the electrode and how it changes with charge and discharge could be analyzed..$^{5,6,10}$

Various preparation methods on $\mathrm{PbO}_{2}$ electrodes were studied in Ref. 11 using X-ray diffraction (XRD). A short washing (less than $1.5 \mathrm{~min}$ ) and drying afterwards leads to the most reliable results.

Specimen preparation is a salient part of the developed method. The aim of this work was to find reproducible and reliable conditions for a preparation method for CLSM analysis. Investigated parameters were duration of the washing process as well as temperature and duration of the drying process in the vacuum drying oven.

\section{Image Acquisition with a Confocal Laser Scanning Microscope}

The examinations were conducted using a Keyence VK-9710 CLSM. The CLSM was placed on a vibration damping table in a glove box providing argon inert gas atmosphere. Thus, oxidation of specimens during acquisition of hundreds of single images for large area scans is precluded.

On the electrodes which were investigated images were acquired at three different locations (top, middle, bottom). Figure 1 shows the directions on an electrode placed under the microscope and the locations of image acquisition. Images were taken at three different heights on the plates (y-direction) each in the middle of the plate in $\mathrm{x}$ direction. The microscope scans the electrode in single slices (distance 


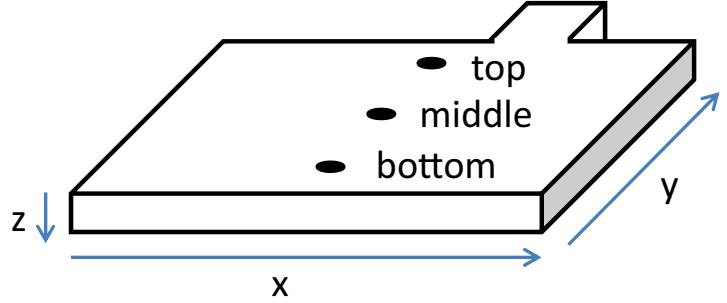

Figure 1. Schematic illustration of directions and locations of image acquisition on a lead-acid battery electrode.

of the slices depending on step sizes in z-direction). These slices are then assembled to one image. During this work, a z-direction range of $200 \mu \mathrm{m}$ was found to be suitable. The step size was set automatically by the microscope according to the variations on the plate (slow changes in z-direction allow large steps, abrupt changes require small steps).

It is possible to either take single images or to run an area scan which is assembled from a number of single images taken in a raster pattern. Figure 2 shows an area scan consisting of 49 single images on the left and two single images taken from the data acquired during the scan on the right. Significant differences in the surface structures in the two single images are visible although the images are located directly next to each other. This has to be kept in mind when analyzing single images.

This fact underlines the importance of scanning larger areas to attain an extensive impression of the surface structure. For this reason, the applicability of the developed method to large surface areas is an important advantage as it is possible without increasing duration and cost of the preparation process. Basically, a complete plate could be scanned. Scan time - increasing virtually linearly with surface area is the only cost parameter that has to be taken into consideration for these area scans. Area scans allow the acquisition of representative images of the investigated surface. Measured surface structures are likely not to be completely covered by few random samples each covering only a small area and spaced widely compared to their area.

As varying surface structures can be very close to each other, it was decided to use only area scans of at least 25 single images. Further on in this work, predominantly single images are shown, but they are chosen representatively from the area scans. As an example of measurement time, an area scan of $0.25 \mathrm{~mm}^{2}$ using the same parameters as used for the majority of scans within this work takes approximately 8 hours.

Complete electrodes can be placed under the microscope. This makes it possible to have a look at various locations on the plate easily, e.g. to analyze influences of inhomogeneous ageing over the plate. To investigate the influence of acid stratification on the electrode surface, acquiring an assembled image in form of a vertical stripe could be an interesting approach as well, which however has not been tested yet.

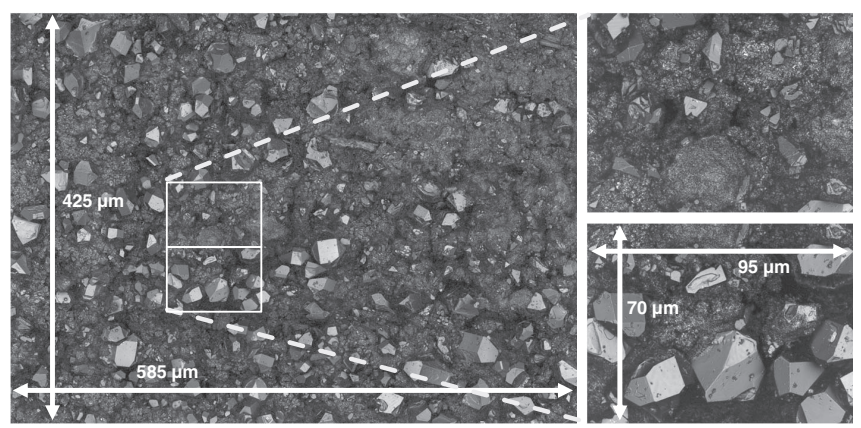

Figure 2. Illustration of a CLSM area scan image consisting of 49 single images (left) and two single images (right) taken from the area scan data. ${ }^{12}$

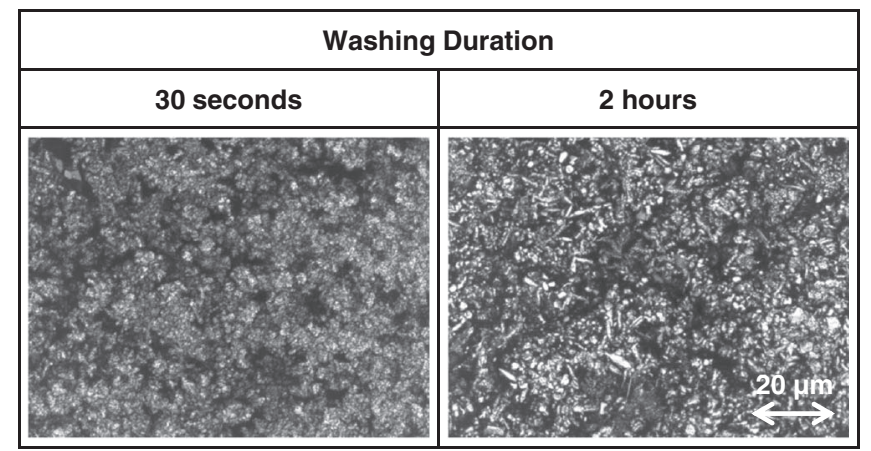

Figure 3. Single CLSM images of two negative electrodes from one battery (same stack) after various washing times.

\section{Specimen Preparation}

For a microscopic analysis, electrodes from lead-acid batteries need to be prepared first. After tear-down of the particular battery, this procedure can be separated into two important steps, which are washing and drying of the electrodes. All electrodes prepared and investigated in this work were taken from the middle of the respective stacks.

Washing duration.- - In a first step the plates ${ }^{\mathrm{d}}$ were washed with tap water to remove electrolyte (sulfuric acid) and to stop (or at least slow down) chemical reactions taking place on the electrodes. Furthermore, the washing step is needed to prevent possible damage of the CLSM equipment.

Various washing durations were examined in order to find an optimal time span for this step. Different middle plates which were removed from the same stack were used for this investigation. Durations of 30 seconds up to $2 \mathrm{~h}$ were tested and compared. For the shortest washing duration of 30 seconds the electrodes were held under a slow water flow in a way that the water ran across every part of the electrodes. This short washing was applied to both sides of the electrodes with a total washing duration of about 30 seconds. For the washing duration of 2 hours a different process was employed. The electrodes were placed in an empty battery case. From a reservoir water was pumped slowly into the housing and flowed back into the reservoir. During the washing process the $\mathrm{pH}$ value was measured without observing noticeable changes.

At the positive electrode the washing duration does not influence electrode structure. For the negative electrode a longer washing duration caused emerging of small and long crystals on a couple of specimens. Figure 3 exemplarily shows this phenomenon. During the longer washing, the electrode surface can react with water and new structures can be built. It is assumed that these new structures are formed through accelerated recrystallization favored by the diluted acid concentration. As well, organic expanders can be part of the negative active mass. If this is the case, they can be removed by longer washing and the surface of the negative electrode can change. ${ }^{13}$

Various battery laboratories suggest washing times of at least $4 \mathrm{~h}$ or more. Sometimes the plates are only washed in a stack which is not sufficient for the complete removal of the electrolyte from the inner side of the plates. For chemical analysis of electrodes (e.g. sulfate content examination) a longer washing duration can be suitable. The main argument for acid-free washing for a longer time is that remaining acid on the plate can still react with the active material and lead-sulfate crystals can grow further.

For optical methods as with a CLSM, a short washing time of 30 seconds was found satisfactory. The electrodes were sufficiently

${ }^{\mathrm{d}}$ A test profile simulating real-world driving conditions including partial state-of-charge (PSOC) control to $80 \%$ SOC was applied to the battery for four months. The battery was opened after the end of the test and the electrodes were taken out for preparation. 


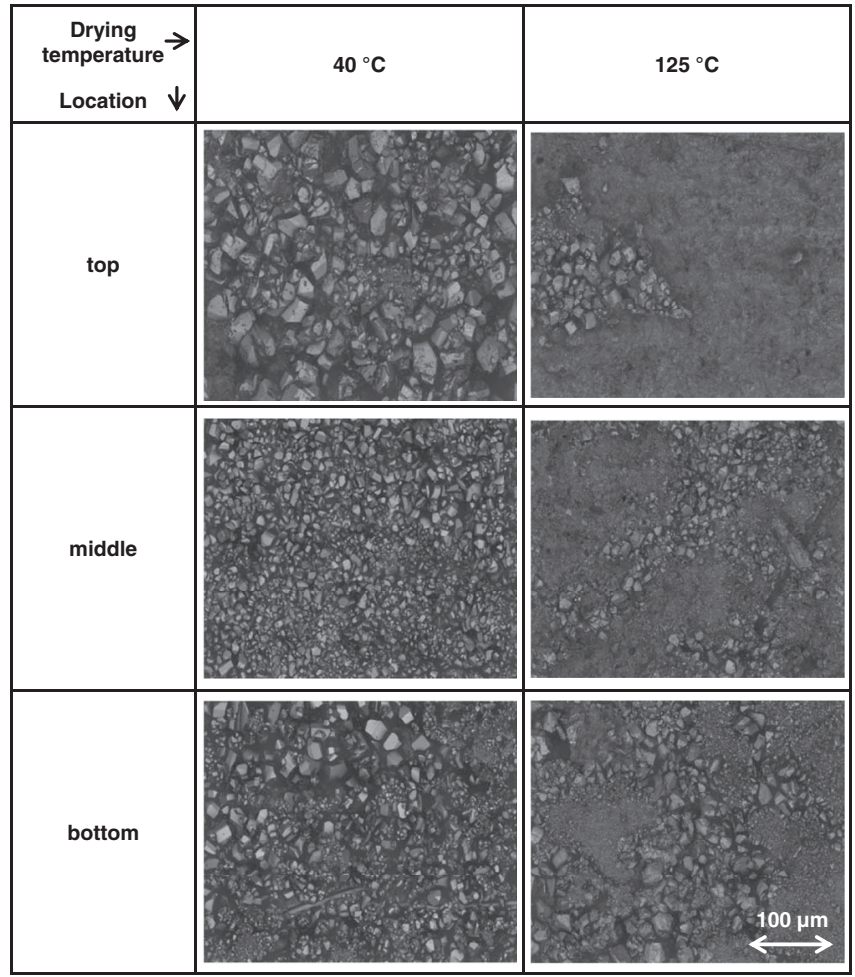

Figure 4. CLSM area scan images at different heights on two positive electrodes from one battery (same stack) dried at different temperatures.

freed from acid for further handling and the original structure of the plates was expected to be kept as unchanged as possible.

Drying temperature.-For drying of the plates, a vacuum drying oven (Binder VDL 115) was used. The parameters of the drying process, duration and temperature, were investigated. Figure 4 shows CLSM area scan images acquired at three locations on two positive middle electrodes from the same stack of a battery ${ }^{\mathrm{e}}$, one dried at $40{ }^{\circ} \mathrm{C}$ for $24 \mathrm{~h}$ and one at $125^{\circ} \mathrm{C}$ for $7 \mathrm{~h}$. When drying at $40^{\circ} \mathrm{C}, 24 \mathrm{~h}$ are needed to ensure a dry specimen. At $125^{\circ} \mathrm{C}$ the drying time can be shorter.

At all three heights differences in the surface structure can be observed. At each height, the surface structures on the plate dried at $125^{\circ} \mathrm{C}$ partially resemble the structures on the one dried at $40{ }^{\circ} \mathrm{C}$, but in some areas they seem to show some significant changes.

It is visible that after drying at $125{ }^{\circ} \mathrm{C}$ there are some parts of the examined electrode that are lost and cannot be analyzed. The high temperature can cause chemical reactions on the electrode surface during drying. After drying specimens at $40{ }^{\circ} \mathrm{C}$ the structure looks more realistic, clear and less inhomogeneous. On the negative electrodes no differences could be observed. Because of the observations made at the positive electrode the lower temperature of $40{ }^{\circ} \mathrm{C}$ was considered appropriate for drying in a vacuum oven, as it provides a well-preserved electrode structure for further optical investigation. Durations of several hours were found sufficient in most cases, 24 hours and more ensure a completely dry specimen.

\section{Experimental}

To verify that the developed method is useful, it was applied to several samples in various states. Charged and discharged state, the influence of different discharge currents and an aged battery from field are investigated.
Charged and discharged state.-For comparison of charged and discharged state, a new flooded OEM SLI 60 Ah battery was used. After initialization with three nominal cycles (charging with $5 I_{20}{ }^{\mathrm{f}}$, $16 \mathrm{~V}$ for $24 \mathrm{~h}$, discharging with $I_{20}$ until $10.5 \mathrm{~V}$ ), the battery was first discharged with $I_{20}$ until $10.5 \mathrm{~V}$, which is equal to $0 \%$ state of charge (SOC). Within $1 \mathrm{~h}$ after finishing the discharge step, two cells were opened. Electrodes from the middle of the stacks were prepared for microscopic examination. The remaining four cells $\mathrm{s}^{\mathrm{g}}$ were charged with $5 I_{20}, 10.67 \mathrm{~V}^{\mathrm{h}}$ for $24 \mathrm{~h}$, which equals $100 \%$ SOC. Again within $1 \mathrm{~h}$ after finishing the charge, two further cells were opened and middle plates were extracted and prepared for further analysis in the same way.

Influence of the discharge current.-To investigate the influence of the discharge current on the electrode structures, the remaining two cells of the battery described in Charged and discharged state subsection under Experimental section were discharged with a higher current rate. The discharge was performed with $4 I_{20}$ until $3.5 \mathrm{~V}^{\mathrm{i}}$, and the battery was opened and middle electrodes were prepared within $1 \mathrm{~h}$ after the end of the discharge. As this discharge current rate is higher, the electrode is at a slightly higher SOC (related to the nominal capacity) compared to the discharge with $I_{20}$ in Charged and discharged state subsection under Experimental section. The reason for this is that the available capacity is decreasing with raising discharge current rate. ${ }^{14}$

Aged battery from field.-As another example of the method, electrodes from an aged battery (approximately five-year-old) which failed cranking in winter were extracted for analysis without any additional conditioning. This means that the SOC of the plates is exactly the same as determined by the usage and field conditions. The open circuit voltage (OCV) was $11.92 \mathrm{~V}$ before opening, so the battery was in a discharged state. Middle electrodes were prepared with the proposed method.

\section{Results and Discussion}

The following various examples show that the described approach can be considered promising for further research. All the images were taken after using the suggested preparation method (30 seconds of washing with tap water and drying at $40{ }^{\circ} \mathrm{C}$ for $24 \mathrm{~h}$ ).

First, electrodes in charged and discharged state are compared. Afterwards, the influence of the discharge current rate is shown. Moreover, images obtained from an aged battery taken from a car after failing cranking of the vehicle in winter are presented.

Charged and discharged state.-To confirm the developed method, surface structures of positive and negative electrodes after charging and discharging (as described in Charged and discharged state subsection under Experimental section) were investigated by the means of CLSM.

The columns on the left and right of Figure 5 depict images of a negative electrode surface in discharged $(0 \%$ SOC after discharging with $\left.I_{20}\right)$ and charged (100\% SOC after charging with $\left.5 I_{20}\right)$ state, respectively. The images are single images obtained by CLSM area scans and represent the structure of the scanned area. At each electrode, area scans were taken at top, middle and bottom of the plates. Figure 6 shows the corresponding images of a positive electrode.

Comparing the two columns in Figure 5, discharged and charged state of the negative electrode clearly show visible differences in surface structure. After charging (right column) a fine-grained pattern

${ }^{\mathrm{f}}$ The current $I_{20}$ is equal to $C_{20} / 20 \mathrm{~h}$, where $C_{20}$ is the nominal capacity.

${ }^{\mathrm{g}}$ The open part of the battery was contacted via a screw into the inter cell connector.

${ }_{\mathrm{h}}^{\mathrm{h}} 16 \mathrm{~V}$ is the voltage limit for charging six cells, $10.67 \mathrm{~V}$ is the corresponding value for charging the remaining four cells.

${ }^{\mathrm{i}} 10.5 \mathrm{~V}$ is the voltage limit for discharging six cells, $3.5 \mathrm{~V}$ is the corresponding value for discharging the remaining two cells. 


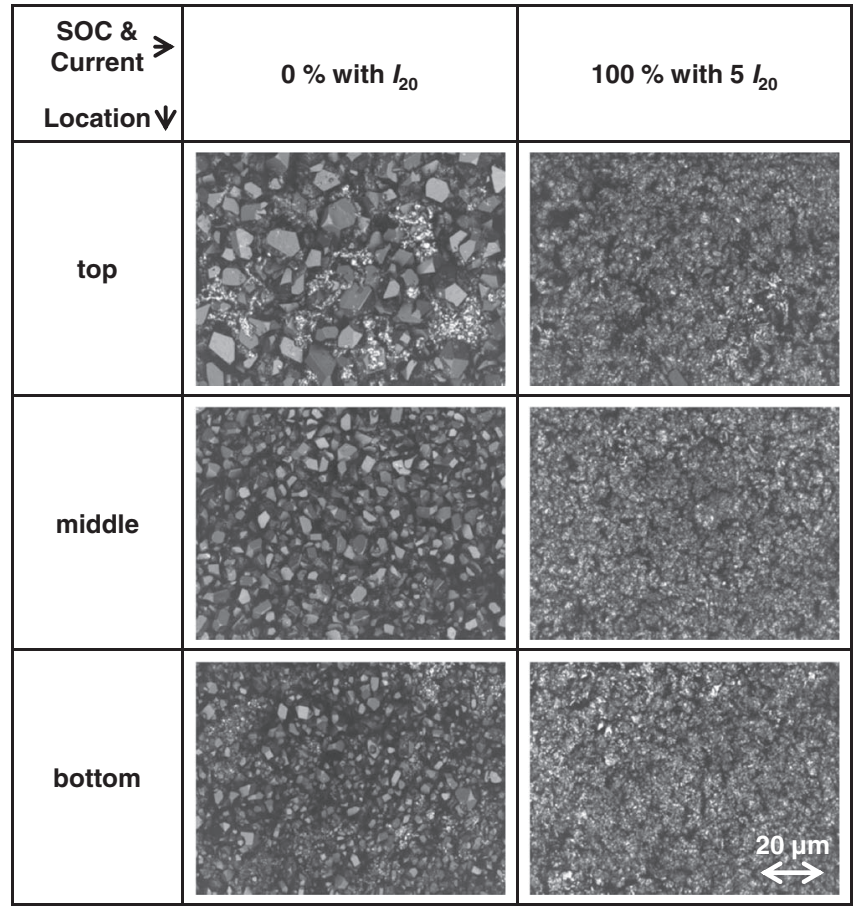

Figure 5. Representative single CLSM images taken from area scans at various heights on negative electrodes after discharge with $I_{20}$ and after full charge.

of lead covers the electrode at all three heights. After discharging (left column) lead-sulfate crystals are all over the surface of the electrode.

For the positive electrode (Figure 6), charging at $5 I_{20}, 2.67 \mathrm{~V}$ per cell with a time limit of $24 \mathrm{~h}$ is not enough to charge the electrode also at the bottom of the plate (right column), visible from some remaining lead-sulfate crystals. If the formation was not complete on these cells, this might be a relict from production, as the battery was new apart from the three nominal cycles for run-in. Only the top and the middle

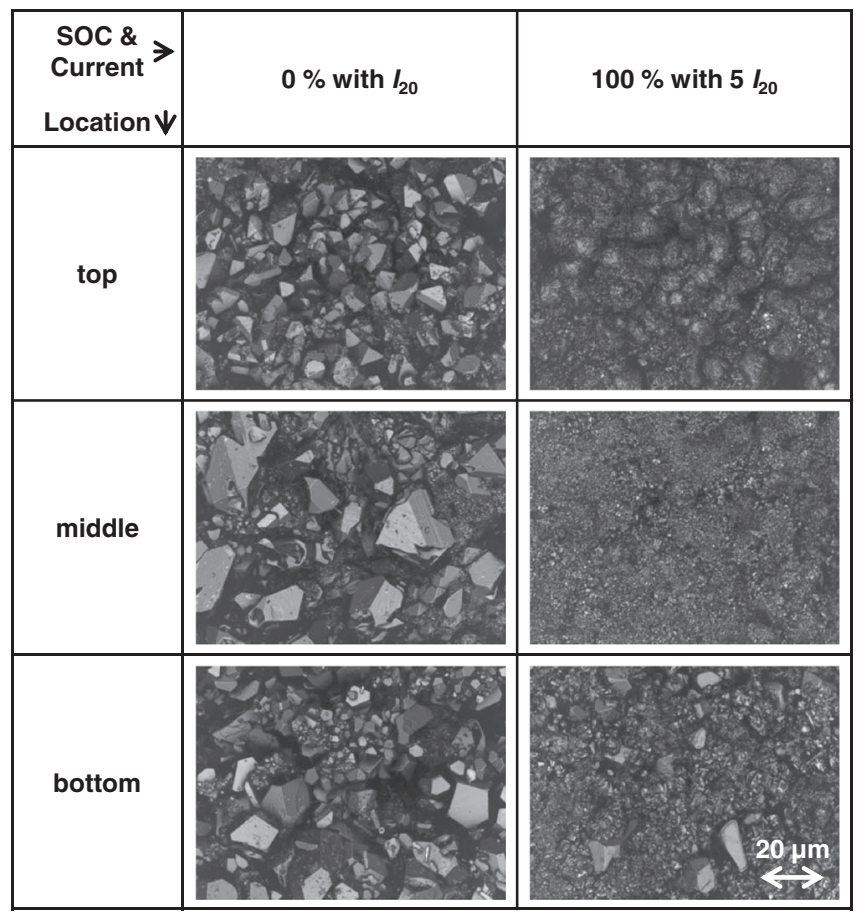

Figure 6. Representative single CLSM images taken from area scans at various heights on positive electrodes after discharge with $I_{20}$ and after full charge. of the plate are charged well. This can also be a result of early acid stratification which was caused by the three nominal cycles. When the plate is discharged (left column), lead-sulfate is covering the surface.

In general, lead-sulfate crystals at the positive electrode are larger compared to the negative electrode. During charging and discharging, the active material at the positive electrode expands, as $\mathrm{PbSO}_{4}$ has a larger volume compared to $\mathrm{PbO}_{2}$. When the battery is charged again, it is not possible to restore the $\mathrm{PbO}_{2}$ to its original small size. In contrast, $\mathrm{Pb}$ at the negative electrode is softer than $\mathrm{PbSO}_{4}$ and can be compressed during discharge when larger $\mathrm{PbSO}_{4}$ is formed. ${ }^{15}$ This is one explanation for the growth of the active material at the positive electrode in general and as well for larger lead-sulfate crystals at the positive electrode. Of course, the pore systems of both types of electrodes offer by far more pore volume for the precipitation of leadsulfate than required during a full discharge to $0 \%$ SOC. The active materials are only partially electro-chemically converted from positive and negative active mass into lead-sulfate and continue to form the conductive backbone of active mass pellets.

Influence of the discharge current.-To check whether noticeable differences on images obtained with the developed preparation method are visible, images after discharge with various current rates (as described in Influence of the discharge current subsection under Experimental section) were compared.

Figures 7 and 8 show single representative CLSM images taken from area scans of negative and positive electrode surfaces acquired at three different heights (top, middle, bottom). The images after discharging with $I_{20}$ (left columns) can be compared with the images after discharging with $4 I_{20}$ (right columns).

At the top of the negative electrodes, number and size of leadsulfate crystals is different (Figure 7). When a discharge starts, first small seed crystals are formed, which then only grow during further discharging. When the discharge current rate is higher, more seed crystals are formed.

A high supersaturation caused by a high current density reduces the particle size as described by Bode. ${ }^{16}$ This can be an explanation why there are more and smaller lead-sulfate crystals at the top of the negative electrode after discharging with $4 I_{20}$.

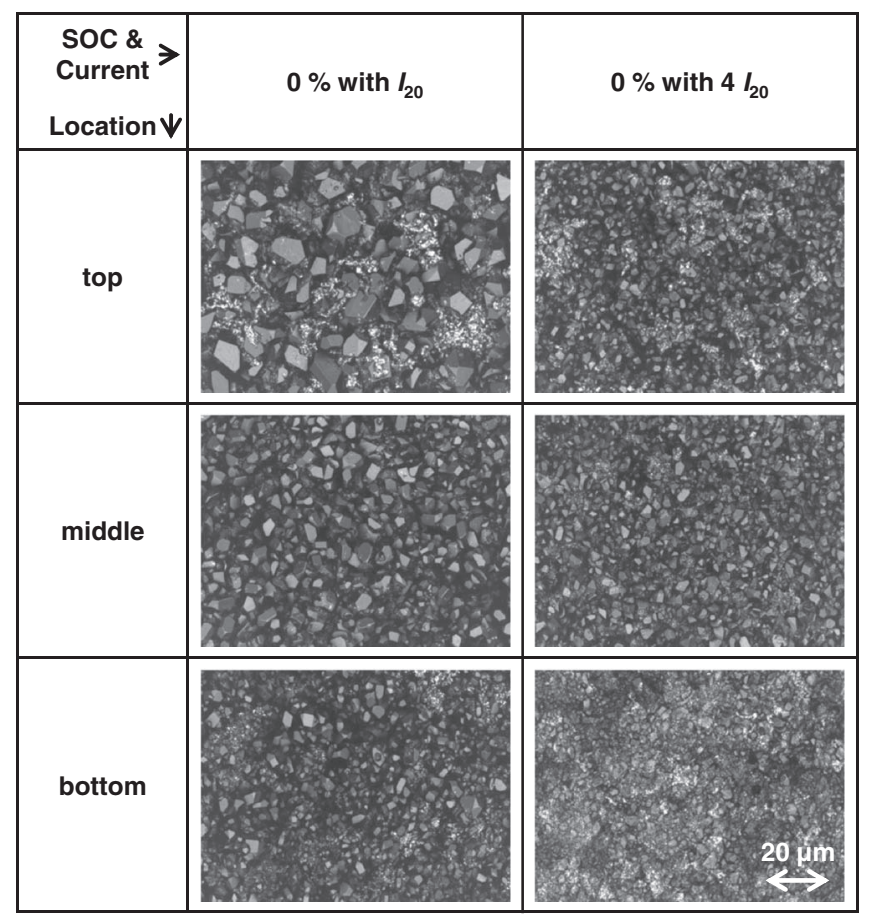

Figure 7. Representative single CLSM images taken from area scans at various heights on negative electrodes after discharge with $I_{20}$ and $4 I_{20}$. 


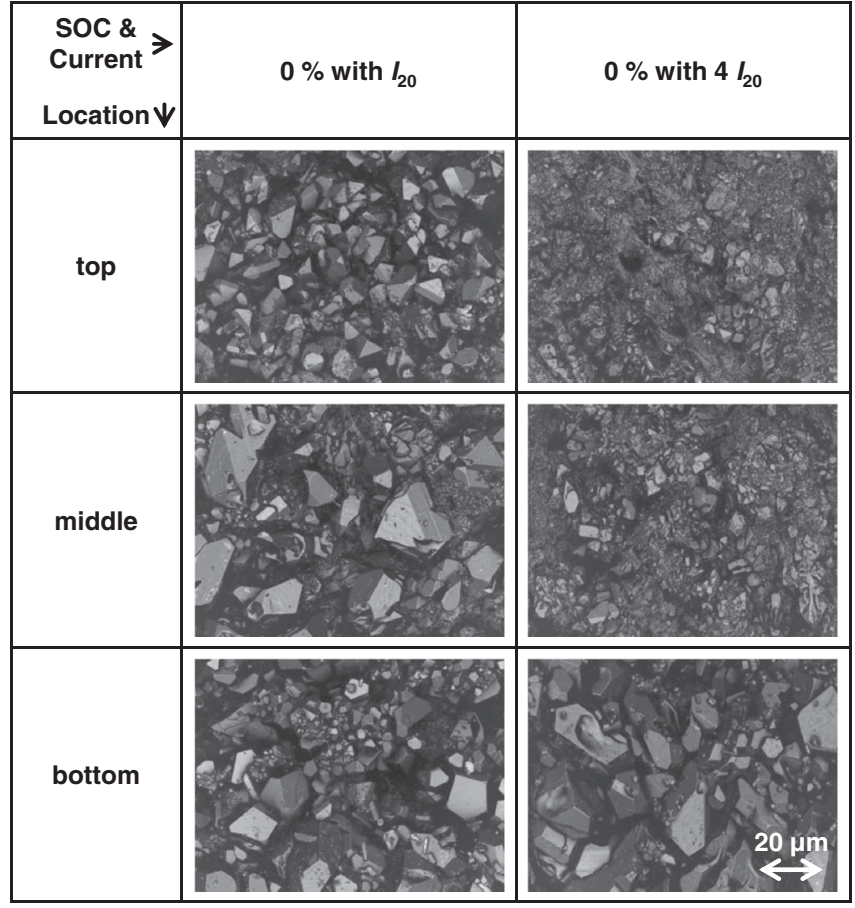

Figure 8. Representative single CLSM images taken from area scans at various heights on positive electrodes after discharge with $I_{20}$ and $4 I_{20}$.

At the bottom, the negative electrode is not completely discharged by the current rate of $4 I_{20}$. The SOC is slightly higher when reaching the end of discharge voltage with the higher current rate of $4 I_{20}$ compared to $I_{20}$. So these images show a good correlation with the theory.

At the positive electrode, smaller lead-sulfate crystals are formed at the top and in the middle of the plate by the discharge with $4 I_{20}$ (Figure 8, right column). During the discharge with $I_{20}$ (Figure 8, left column), crystals are formed at every height of the plate.

Comparing the three locations in Figure 8 after discharge with $4 I_{20}$ (right column), most crystals are visible at the bottom. The reason for this could be that during the full charge in the step before discharging with $4 I_{20}$, crystals at the bottom were not completely dissolved (Figure 6, right column).

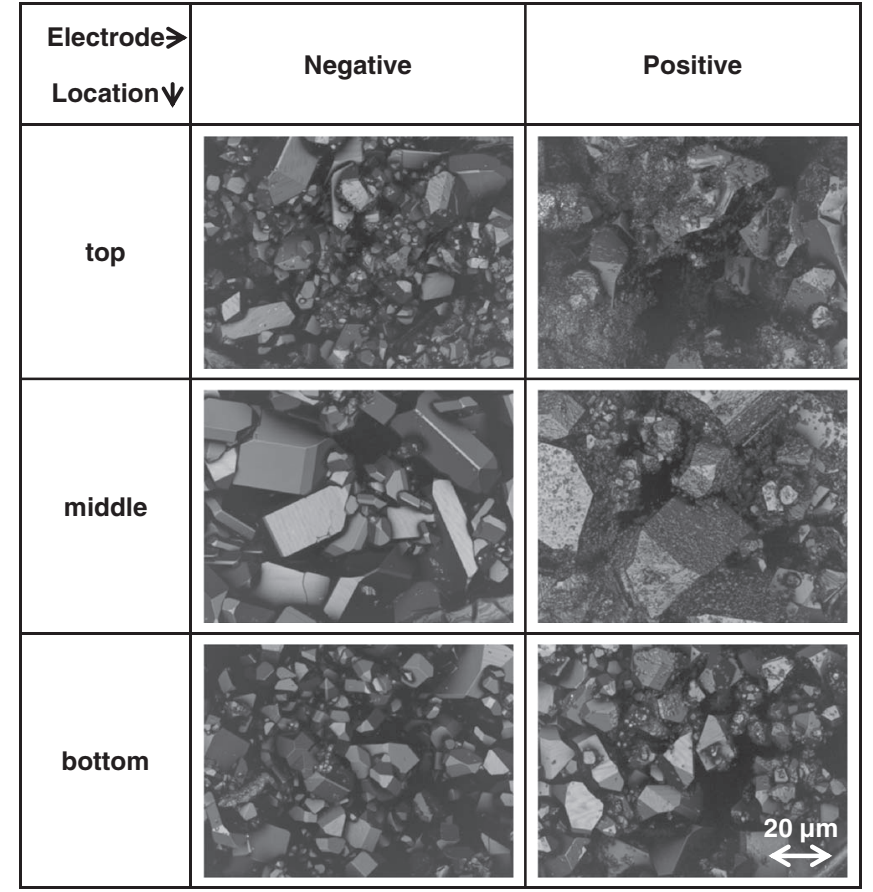

Figure 9. Representative single CLSM images taken from area scans at various heights on a negative and positive electrode from an old battery failing cranking in winter.

It is possible to show clear differences on both electrode surfaces between various discharge current rates with the presented preparation method.

Aged battery from field.-For further evaluation of the method an aged battery (state as described in Aged battery from field subsection under Experimental section) from the field failing cranking in winter was examined.

Figure 9 shows electrode surfaces of a negative and positive plate. Presented images are representative single CLSM images chosen from area scans acquired at three heights. One can clearly notice how big crystals have grown and observe smaller structures on the bigger crystals.

Figure 10 shows a CLSM area scan of a positive electrode from the same battery as shown in Figure 9. It was acquired in the middle of

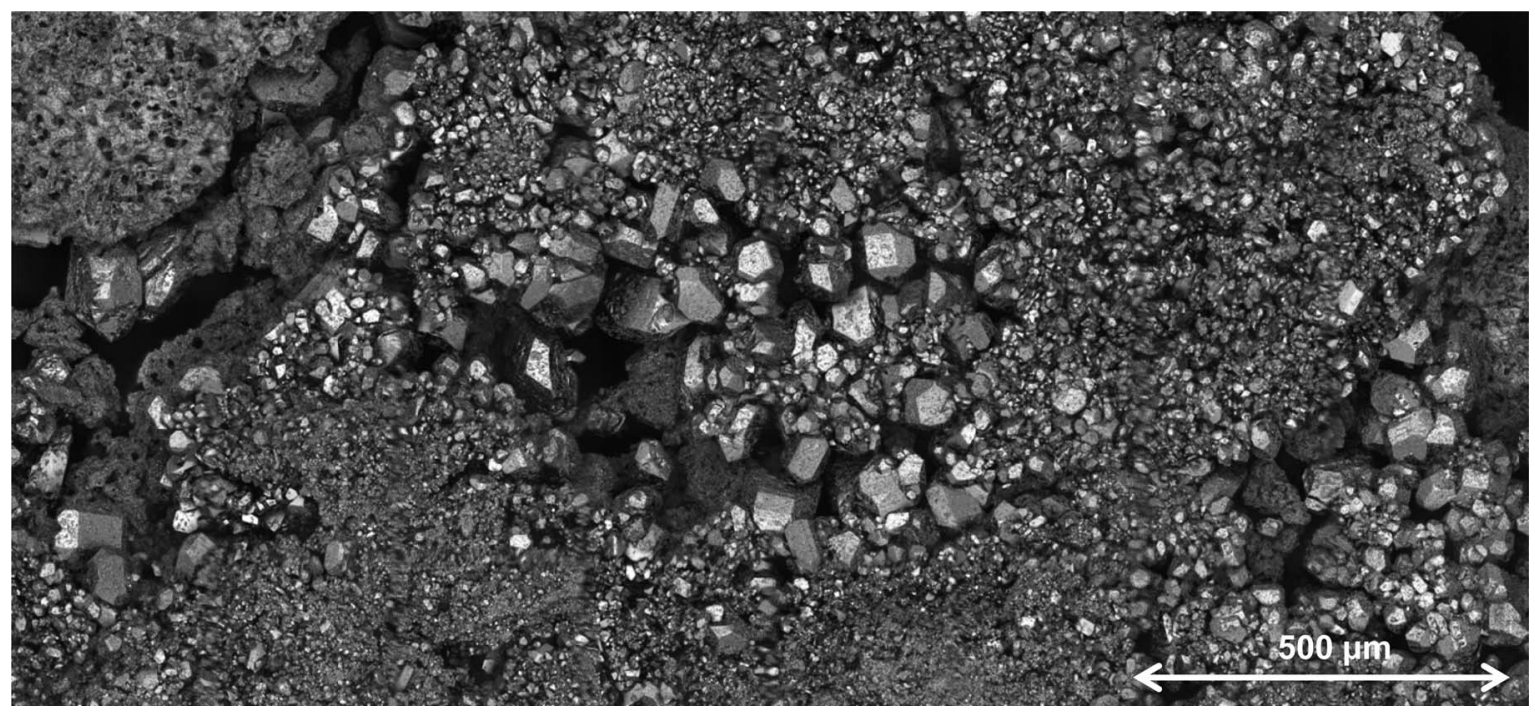

Figure 10. CLSM area scan image taken at center of a positive electrode from an old battery failing cranking in winter. 
the electrode and is assembled from 40 single images taken in a raster pattern. In the covered area of about $1.8 \mathrm{~mm}^{2}$, strong spatial variations in the surface structure can be observed. There are bigger crystals in the middle of the image and at several other locations. Smaller and medium-sized crystals can be found on bottom and top of the image. Furthermore, a structure looking similar to a sponge can be found in the upper left corner. Again, it shows how insightful scans obtained using the presented approach on large areas can be.

\section{Summary}

Preparation methods for the optical analysis of surface structures of lead-acid batteries were investigated. A fast and simple method was developed and tested. The main focus was laid on finding a suitable procedure for the specimen preparation (duration of washing, temperature and duration of drying). The developed method employs a short washing process (30 seconds) and drying in a vacuum drying oven $\left(40^{\circ} \mathrm{C}\right.$ for $\left.24 \mathrm{~h}\right)$ followed by CLSM imaging.

The applicability of the method has been shown on several images acquired from various specimens in different states. Charged and discharged states of the electrodes show clear differences in the amount of lead-sulfate crystals. Discharging with a higher current rate in respect to the nominal current causes formation of smaller crystals. Analyzing an aged battery from field, larger crystal growth could be shown.

The fact that the method can easily be adapted to obtain images of large areas and, thus, provides representative insight into spatially varying surface structures makes it a promising approach for further research and application. In the future detailed investigations on reactions during discharge and charge, including dynamic charge acceptance will be performed. Another possible application of the method is the observation of cross-sectional areas of electrodes to investigate thickness changes related to SOC or ageing. Further, images by CLSM can be compared with XRD for a deeper understanding of the surface structure.

\section{Acknowledgments}

We thank Dr. Eckhard Karden (Ford Research and Advanced Engineering Europe, Aachen), Prof. Dr. Julia Kowal (TU Berlin, formerly ISEA, RWTH Aachen University), Julia Badeda and Grzegorz Pilatowicz (both ISEA, RWTH Aachen University) for useful discussions. The support of Jens Münnix and Marc Zimmermann (both ISEA, RWTH Aachen University) in the laboratory is appreciated.

\section{References}

1. Y. Yamaguchi, M. Shiota, M. Hosokawa, Y. Nakayama, N. Hirai, and S. Hara, J. Power Sources, 102, 155 (2001).

2. Y. Yamaguchi, M. Shiota, Y. Nakayama, N. Hirai, and S. Hara, J. Power Sources, 93, 104 (2001).

3. N. Hirai, S. Kubo, and K. Magara, J. Power Sources, 191, 97 (2009).

4. M. Shiota, Y. Yamaguchi, Y. Nakayama, K. Adachi, S. Taniguchi, N. Hirai, and S. Hara, J. Power Sources, 95, 203 (2001).

5. D. Pavlov and E. Bashtavelova, J. Electrochem. Soc., 133, 241 (1986).

6. D. Pavlov and S. Ignatova, J. Appl. Electrochem., 17, 715 (1987).

7. D. Pavlov, T. Rogachev, P. Nikolov, and G. Petkova, J. Power Sources, 191, 58 (2009).

8. Z.-i. Takehara, J. Power Sources, 85, 29 (2000).

9. D. Pavlov, P. Nikolov, and T. Rogachev, J. Power Sources, 196, 5155 (2011).

10. K. Micka, M. Svatá, and V. Koudelka, J. Power Sources, 4, 43 (1979).

11. T. Laitinen, G. Sundholm, and J. K. Vilhunen, J. Power Sources, 32, 71 (1990).

12. H. Budde-Meiwes, C. Rahe, J. Kabzinski, J. Kowal, D. Sauer, and E. Karden, Impact of microscopic electrode structure on dynamic charge acceptance considering short-term history, current and temperature, 13th European Lead Battery Conference (ELBC), Paris, France, 2012.

13. Herbert Borowski. personal communication, February 2013.

14. A. Jossen and W. Weydanz, Moderne Akkumulatoren richtig einsetzen, 1st edition, Ubooks Verlag, Neusäß, 2006, ISBN: 3-937536-01-9.

15. D. A. J. Rand, P. T. Moseley, J. Garche, and C. D. Parker, editors, Valve-Regulated Lead-Acid Batteries, 1st edition, Elsevier, 2004, ISBN: 0-444-50746-9.

16. H. Bode, Lead-acid batteries, John Wiley \& Sons, 1977, ISBN: 0-471-08455-7. 